\title{
Performance Analysis of Existing Location Management Scheme with its Variants in PCS Network
}

\author{
Rajeev R. Kumar Tripathi \\ SSSIST \\ Sehore (M.P) India
}

\author{
G.S. Chandel \\ SSSIST \\ Sehore (M.P) India
}

\author{
Ravindra Gupta \\ SSSIST \\ Sehore (M.P) India
}

\begin{abstract}
This paper is presenting a comparative study of existing location management scheme with its various variants in Personal Communication Services (PCS) network. The existing location management scheme is based on two tier database architecture; Home Location Register (HLR) and Visitor Location Register (VLR).In the existing location management scheme, there is a single HLR. In the HLR, profile of a Mobile Terminal (MT) is permanently stored. The VLR caches the profile of the MT from the HLR when it is providing the service to the mobile terminal. Mobile terminal, on move changes its location and comes under the Registration Area (RA) of new VLR. The MT profile should be removed from the VLR from where this MT is coming from and the HLR should have the information of current location of the MT. In the existing location management scheme this is done by the explicit deregistration scheme. In the existing call delivery procedure of PCS network, the HLR is always consulted to get the current location of the MT. This call delivery procedure supports an idea that is it necessary to inform the VLR that MT who was residing in its RA is now in the RA of another VLR? This paper has used other de-registration schemes as: Implicit deregistration, Polling de-registration, Timeout de-registration scheme and Group de-registration scheme in the variant of existing location management scheme. The single HLR architecture is prone to bottleneck and multi HLR architecture for PCS network is proposed for the efficient call delivery. Analysis done in this paper shows that we can significantly reduce the location management cost in the various variants of the existing location management schemes. This paper finally concludes that group de-registration scheme when implemented with the proposed multi HLR architecture gives better results.
\end{abstract}

\section{Keywords}

Home location register, Visitor location register, Registration area, Explicit de-registration, Implicit de-registration scheme, Polling de-registration scheme, Timeout de-registration scheme, Group de-registration scheme, Multi HLR architecture.

\section{INTRODUCTION}

In the existing location management scheme in PCS network has two tier database architecture containing: Home Location Register (HLR) and Visitor Location Register (VLR). The existing location management scheme has a single HLR and many VLRs. In PCS network the smallest coverage area is termed as cell. Each cell has a Base Station (BS). Registration area may have tens or hundreds of cells. In PCS network on requirement Base Controller System (BSC) may be introduced to control a group of BSs [11]. The VLR can serve one or more RAs. A model of PCS network is shown in the figure 1 . Functional components of PCS network are connected by various switches (Signal Transfer Switch: STP and Signaling System-7: SS7 switches). These switches work as an interface as functional components are vendor dependent. Mobile Switch Center (MSC) is collocated with VLR. When an MT changes its location from one RA to another RA, the MT sends a

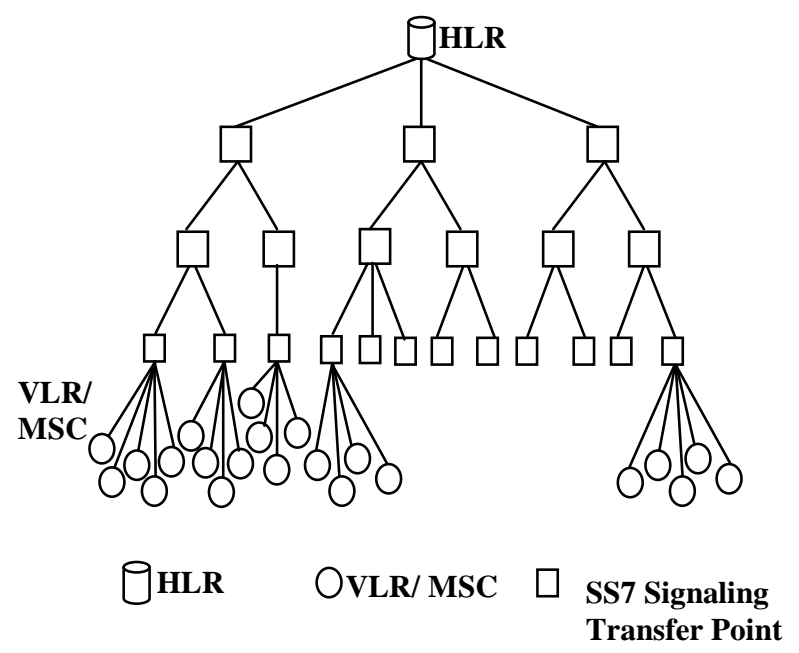

Fig1: Analytical model of PCS network

location registration request to the VLR through BS. The VLR registers the MT and informs the HLR about this change by sending the registration message. On reception of this message HLR updates the current location of the MT. The HLR now sends a registration cancellation message to the old VLR. This message is sent to the VLR to remove the entry of the left MT. This process is necessary as stale entries occupied the space of VLR database. On reception of the location cancellation message the old VLR remove the entry of the left MT and acknowledges the HLR. Finally the HLR sends the acknowledgement to the new VLR. This entire process is termed as location registration $[1,2,3]$. Location registration is being shown in the figure 2 .

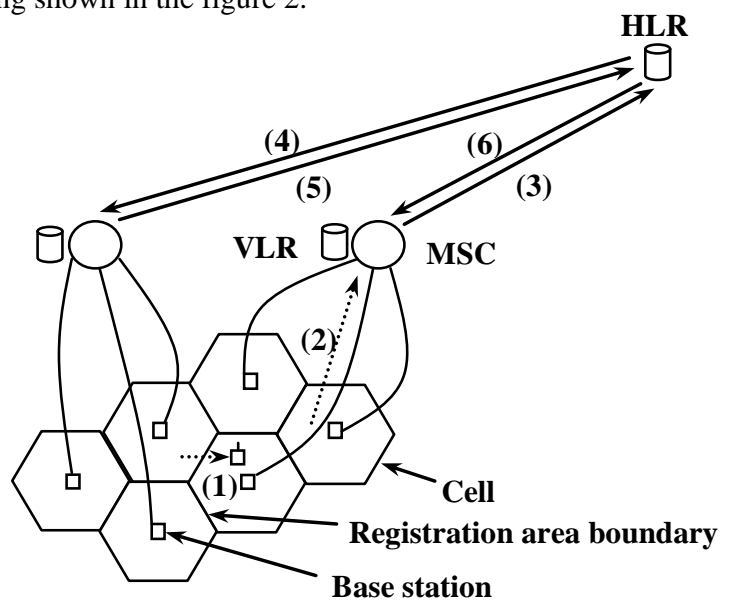

Fig 2: Location registration 
Steps involved in the location registration process are as:

Step 1: On movement, the MT changes its RA, and comes in a new RA being served by a new VLR. The MT detects it and sends a location registration message to the nearest BS.

Step 2: The BS sends this message either to the BSC (if BSC is present in the PCS network) or to the MSC/VLR directly.

Step 3: The new MSC/VLR updates this information in the VLR. This update shows that the MT is now in the RA of this VLR. This change in the location of MT should be informed to the HLR so that in case of an incoming call to this MT, the HLR should provide a routable path. The VLR sends a registration message to the HLR.

Step 4: The HLR on reception of this message sends a registration acknowledgment message to the new MSC/VLR together with a copy of the subscriber's user profile. This information is used to validate the subscriber at new MSC/VLR end.

Step 5: As the MT is coming from a VLR (say old VLR) to the new VLR (the MT is currently residing in), the HLR sends a registration cancellation message to the old MSC/VLR to remove the MT's information from the old VLR. This is being done to keep the PCS network free from inconsistent information of an MT.

Step 6: The old MSC/VLR deletes the profile of the mobile terminal at its associated VLR and sends a cancellation acknowledgment message to the HLR.

In location management, databases are updated thrice as:

a) At new VLR, when MT is coming in the RA of this.

b) At the HLR, when new VLR sends the location update.

c) At the old VLR, when the HLR sends registration cancellation.

Location management cost will increase in the same proportion to the database access. More database access, more cost incurred into the location management.

Call delivery process has the following steps:

Step 1: When an MT initiates a call, the call initiation signal is sent to the serving MSC by the BS of the cell in which the MT is residing. The call initiation signal may also be sent to the serving MSC by a nearest BS if the MT is at boundary of two cells.

Step 2: The MSC of the calling mobile terminal sends a location request message to the HLR to get the current location of the mobile terminal.

Step 3: The HLR determines the current serving MSC of the called mobile terminal and sends a route request message to this MSC.

Step 4: The MSC determines the cell location of the called mobile terminal and assigns a Temporary Location Directory Number (TLDN) to the called mobile terminal. The MSC then sends this TLDN to the HLR.

Step 5:The HLR sends the TLDN to the MSC of the calling mobile terminal. The calling MSC can now set up a connection to the called MSC through the PSTN.

In the call delivery process, the HLR is being queried to get the current location of the MT. This fact supports an idea of implicit de-registration. This scheme is being discussed in the next section.

\section{IMPLICIT DE-REGISTRATION SCHEME}

In this scheme when an MT enters into the new RA, it sends a location registration message to the new MSC/VLR. The new MSC registers this MT in the VLR. This change in formation of the MT is reported to the HLR. The HLR updates the location of the MT and sends an acknowledgement to the new VLR with the MT profile. In [1] this scheme is termed as modified HLRVLR location management scheme. In this scheme less cost is incurred in location management as only two databases are accessed as: 1) Database of HLR ,2) database of new VLR. This scheme is cost efficient than the explicit de-registration scheme. But this scheme suffers from problem of stale entries of left MTs at VLR ends. To remove the stale entries from the VLR end some de-registration schemes were proposed. In the next section these de-registration schemes are being discussed.

\section{DE-REGISTRTAION SCHEMES TO REMOVE STALE ENTRIES FROM THE VLR}

This section is shading light on four de-registration schemes as: polling de-registration scheme and timeout de-registration scheme, movement based de-registration scheme and distance based de-registration scheme.

\subsection{POLLING DE-REGISTRATION SCHEME:}

In polling de-registration scheme, the VLR periodically sends a polling signal in its RA through BSC and BS (sometimes BS is termed as BTS: Base Transceiver System). The MTs, present in the RA, send acknowledgement back to the VLR. In this way the VLR decides that how many MTs are still in its RA. Entries of the MTs, who have not sent acknowledgement, are removed from the VLR by assuming that MTs have left the RA. Polling process appears as if the network has an incoming call to be delivered to the MT. Polling process is shown in the figure $3[2]$.

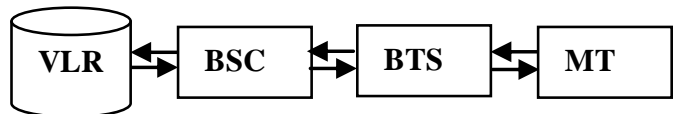

Fig 3: Polling de-registration scheme

\subsection{TIMEOUT DE-REGISTRATION SCHEME}

Timeout de-registration is a refinement of polling deregistration scheme. In this scheme, the MT has to periodically send registration message to the VLR. On the basis of received registration messages, the VLR decides that which MT is still in its RA and which one has left. This scheme is a refinement of polling de-registration scheme because in this scheme we have just half reduced the total cost in comparison to the polling deregistration process. Timeout de-registration is shown in the figure $4[2]$.

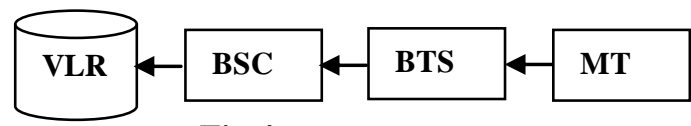

Fig 4: Timeout de-registration 


\subsection{MOVEMENT-BASED DE- REGISTRATION}

In this scheme a movement threshold is defined on the basis of number of movement across cell boundary. If an MT reaches this threshold, the MT performs location updates. This scheme is shown in the figure 5 .

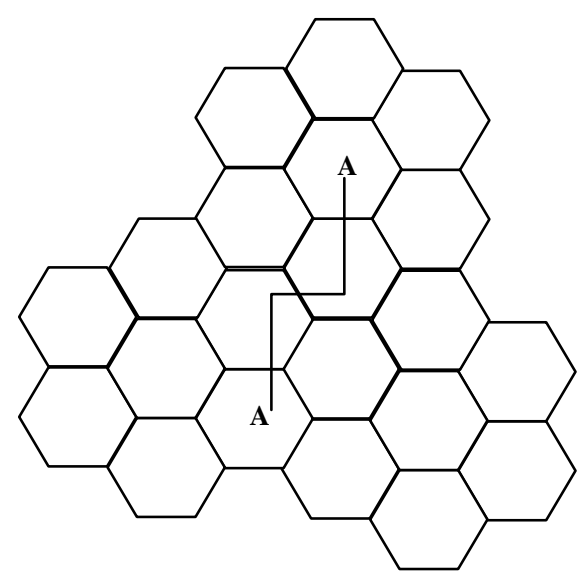

Fig5: Movement based de-registration scheme

\subsection{DISTANCE-BASED DE-}

REGISTRATION: In this scheme a distance threshold is defined between two cells: this distance is counted from cell one from where last location update was made by the MT to another cell. When the MT reaches this distance threshold, it again performs the location update. This scheme is shown in the figure 6.

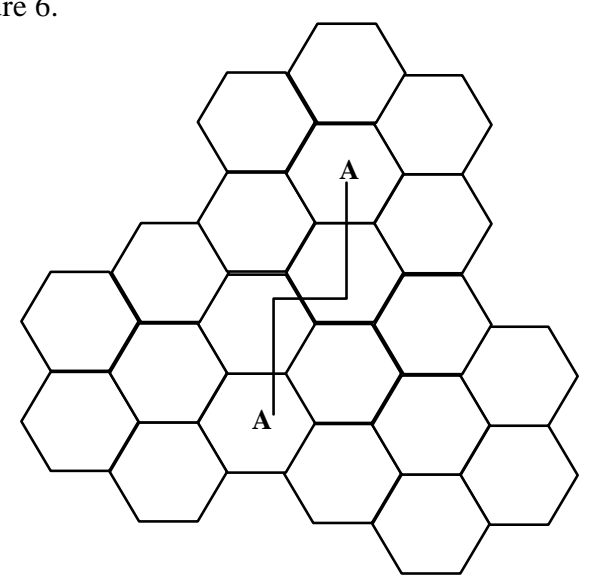

Fig 6: Distance based de-registration scheme

The performance of movement based and distance based deregistration schemes are evaluated by Nov and Sidi [8] on a simplified one-dimensional movement model. Result shows that the distance-based scheme produces the best performance, but its implementation incurs the highest overhead. Due to the overhead involved in these two schemes we have not covered these two schemes in this paper.

Timeout de-registration scheme is more efficient than polling de-registration scheme as in timeout, signaling cost from MT to VLR is just half than the polling. These two schemes appear suitable for the removal of stale entries from VLR, however the both schemes seriously suffer from the problem of synchronization. In next section this problem is being discussed.

\section{PROBLEM OF SYNCHRONIZATION}

In polling and timeout de-registration schemes, network comes to know about the real status of an MT after a time, i.e. whether the MT is in its RA or not. Network decides when to invoke the process of either polling de-registration or timeout deregistration. Let this period is $\Delta t$. If an MT changes its location just after $\Delta t$ then its entry will remain in the VLR database till the next $\Delta \mathrm{t}$. However MT is now in other RA. We are calling this problem as problem of synchronization. If MT leaves the RA just before $\Delta t$, these two schemes are good enough to remove the stale entries from VLR. The MT's movement is quietly unpredictable hence no one can guarantee about this. Here removal of stale entries of MTs is time based. Z. Mao suggests a novel approach of de-registration called group deregistration scheme. In group de-registration scheme, removal of stale entries are based on movement not on time.

\section{GROUP DE-REGISTRATION SCHEME}

In this scheme HLR maintains Old Mobile List (OML) for each VLR. Whenever an MT from one VLR comes in RA of another VLR, HLR keeps the MT's profile into the OML of the VLR from where MT is coming. When a registration update is received by the HLR from this VLR, HLR sends the OML of this VLR with the acknowledgement.

If $\mathrm{MT}_{1}$ leaves $\mathrm{VLR}_{1}$ and joins $\mathrm{VLR}_{2}, \mathrm{VLR}_{2}$ registers this $\mathrm{MT}_{1}$ at its side and informs HLR about the current location of $\mathrm{MT}_{1}$. The HLR puts the MTinformation into OML of VLR $\mathrm{VR}_{1}$ and sends an acknowledgement to $\mathrm{VLR}_{2}$ along with the OML of $\mathrm{VLR}_{2}$ along with the OML of $\mathrm{VLR}_{1}$. Whenever HLR receives any location update message by $\mathrm{VLR}_{1}$, HLR sends the OML of $\mathrm{VLR}_{1}$ with an acknowledgement and $\mathrm{VLR}_{1}$ knows that $\mathrm{MT}_{1}$ has left its RA. In this scheme de-registration of left MTs are being performed on movement basis not on time basis. This is reason the why this scheme is used in the proposed multi HLR architecture against to the explicit de-registration scheme $[2,3$, 4].

\section{TYPES OF MOVE DEFINED FOR AN}

MT: During movement, an MT may change the VLR. If the MT comes to the new RA which is still being served by the same VLR, this change in location is only updated to the VLR not at the HLR. On the other hand if VLR is changed, location registration process is initiated. In this paper we have defined two basic movement of MT as [1-5]:

6.1. Intra-VLR Move: In this type of movement, the MT performs the movement within the RA of same VLR. In this case, MT's current location information is updated in the VLR. This move does not affect the HLR.

6.2. Inter-VLR Move: This move occurs when MT changes its RA and new RA is being served by the new VLR. When this move occurs, location registration process is initiated and HLR is reported about this change. The new VLR makes the entry of MT's profile in its database and the old VLR removes the profile of this left MT.

Intra-VLR move incurs less cost in comparison to the interVLR move as in intra-VLR move only the serving VLR is updated but in the inter-move three databases are updated. . 


\section{MULTI HLR ARCHITECTURE}

To remove the bottleneck from the HLR, we are proposing an idea of multi HLR architecture. This multi HLR architecture is still a centralized approach [3]. In this architecture, we are separating the HLR zone wise or circle wise. We have classified the entire coverage area of the networks either in zone wise or in circle wise. In each zone or circle, we have one HLR. Conceptually each zone or circle is treated as if it is entire network. This HLR may serve many VLRs. It architecture is same as shown in the figure 2 . In this proposed architecture we have two types of HLR: Resident-HLR and Serving-HLR. Resident-HLR is the HLR where the MT often resides. The profile of MT is permanently stored at this HLR. On move, the MT may change the HLR. Serving-HLR is the HLR where the MT resides when it leaves the circle or zone. It is a case of roaming. In this proposed architecture we have the following types of move described as $[3,10]$ :

\subsection{INTRA-VLR-RESIDENT-HLR MOVE}

When this move occurs, the MT changes its RA but the serving VLR remains same. This move is shown in the figure 7. The MT moves from $\mathrm{RA}_{1}$ to $\mathrm{RA}_{2}$ but the serving $\mathrm{VLR}_{1}$ is same. In this type of move only $\mathrm{VLR}_{1}$ is updated. In this move, the MT is in its resident-HLR.

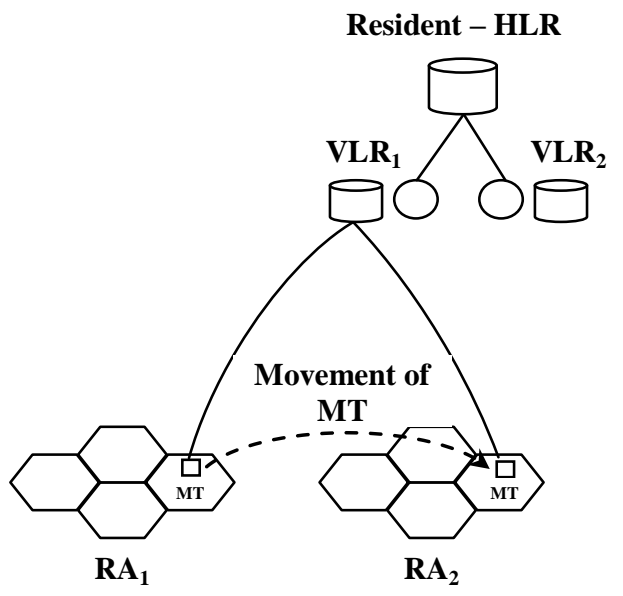

Fig 7: Intra-VLR-Resident-HLR Move

\subsection{Intra-VLR-Serving-HLR Move}

When this move occurs, the MT changes its RA but the serving VLR remains same. This move is shown in the figure 8 . The MT moves from $\mathrm{RA}_{1}$ to $\mathrm{RA}_{2}$ but the serving $\mathrm{VLR}_{1}$ is same. In this type of move only $\mathrm{VLR}_{1}$ is updated. In this move the MT is in roaming i.e. outside the circle and it is being served by serving-HLR.

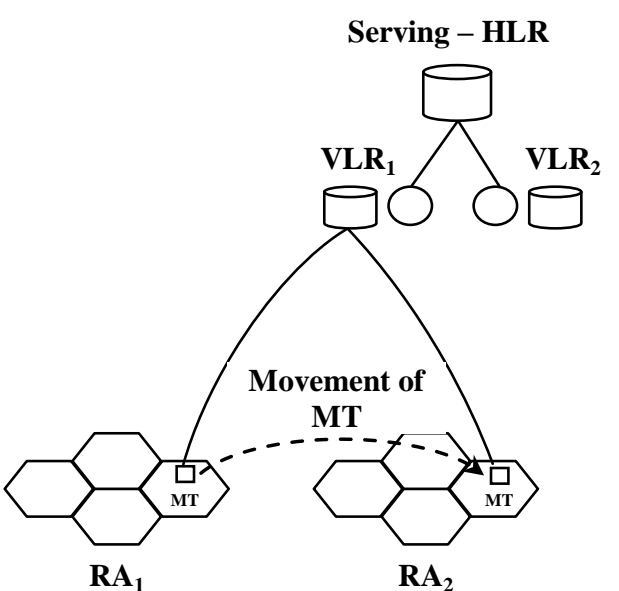

Fig 8: Intra-VLR-Serving-HLR move

\subsection{Inter-VLR-Resident-HLR Move}

When this move occurs, the MT changes its RA along with the VLR. This move is shown in the figure 9. The MT moves from $R A_{1}$ to $R A_{2}$. The $R A_{1}$ is being served by the $V_{L R}$ and $R A_{2}$ by the $\mathrm{VLR}_{2}$. This move is taking place in the resident-HLR region. In this move, the location registration process of IS-41 is initiated and the MT is registered at the $\mathrm{VLR}_{2}$, the residentHLR is updated for this change and the old VLR, VLR 1 is informed to de-register the MT's profile.

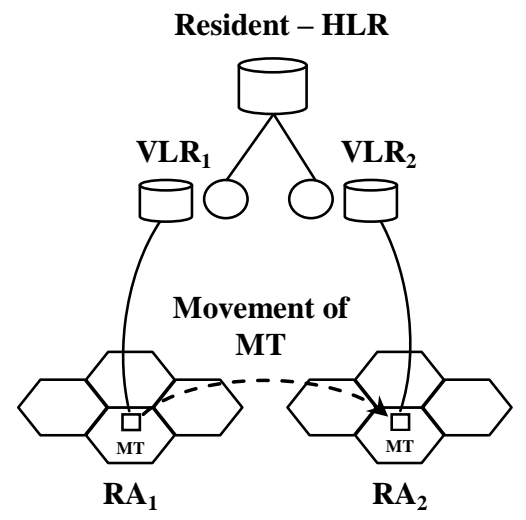

Fig 9: Inter-VLR-Resident-HLR move

\subsection{Inter-VLR-Serving-HLR Move}

When this move occurs, the MT changes its RA along with the VLR. This move is shown in the figure 10. The MT moves from $\mathrm{RA}_{1}$ to $\mathrm{RA}_{2}$. The $\mathrm{RA}_{1}$ is being served by the $\mathrm{VLR}_{1}$ and $\mathrm{RA}_{2}$ by the $\mathrm{VLR}_{2}$. This move is taking place in the serving-HLR region (outside the circle or zone, it is a case of roaming). In this move, the location registration process of IS-41 is initiated and the MT is registered at the $\mathrm{VLR}_{2}$, the serving-HLR is updated for this change and the old VLR, VLR 1 is informed to de-register the MT's profile. The resident-HLR remains unchanged. 


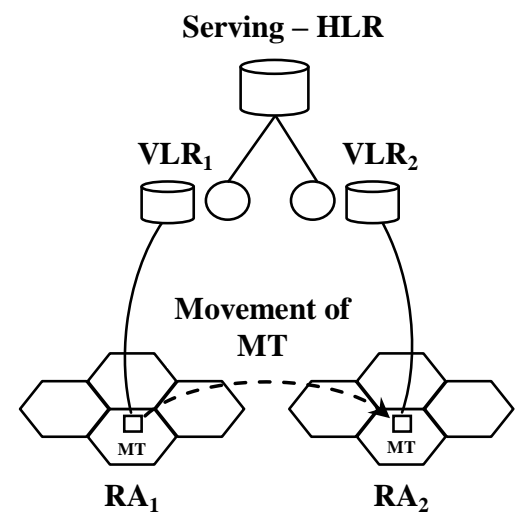

Fig 10: Inter-VLR-Serving-HLR move

\subsection{Inter-VLR-Inter-HLR Move}

In this type of move HLR is changed. This type of move occurs into three cases.

\subsubsection{MT moves from resident-HLR to serving-HLR}

This type of move is shown in the figure 11. The MT is currently residing in the $\mathrm{RA}_{3}$, this $\mathrm{RA}_{3}$ is being served by the $\mathrm{VLR}_{3}$. The $\mathrm{VLR}_{3}$ is being served by the resident-HLR. The MT is currently residing in its resident- HLR. On move, the MT changes the RA and comes in the $\mathrm{RA}_{2}$. The $\mathrm{RA}_{2}$ is being served by the VLR $\mathrm{VL}_{2}$ This $\mathrm{VLR}_{2}$ is being served by the serving-HLR i.e. the MT is in roaming. In this move, the MT initiates the location registration process. Registration of the MT takes place at $\mathrm{VLR}_{2}$. The $\mathrm{VLR}_{2}$ informs the serving-HLR. The servingHLR determines the resident-HLR of the MT and sends a location registration update to the resident-HLR indicating that the MT is now in the circle of this HLR. The resident-HLR updates this location of MT in the database. The resident-HLR sends registration cancellation message to the $\mathrm{VLR}_{3}$. The $\mathrm{VLR}_{3}$ removes the profile of the MT and sends an acknowledgment to the resident-HLR. The resident-HLR sends back an acknowledgement to the serving-HLR with the MT profile. Finally the serving-HLR sends back an acknowledgement to the $\mathrm{VLR}_{2}$.

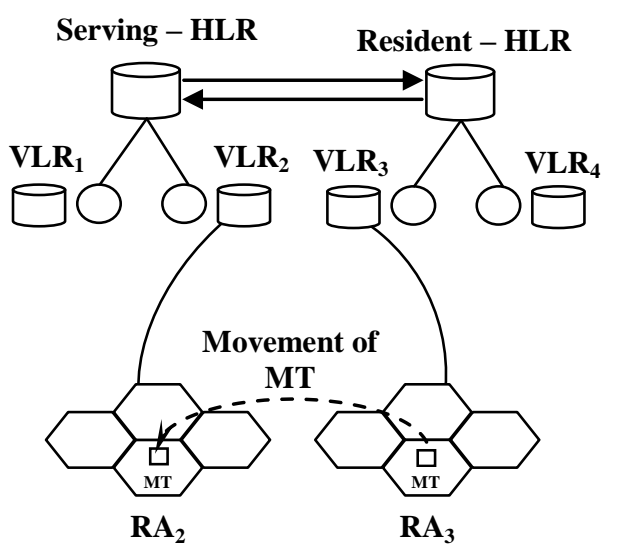

Fig 11: Inter-VLR-Inter-HLR move (from resident-HLR to serving-HLR)

\subsubsection{MT moves from serving-HLR to resident-HLR}

This type of move is shown in the figure 12. The MT is currently residing in the $\mathrm{RA}_{3}$, this $\mathrm{RA}_{3}$ is being served by the $V_{L R}$. The $V_{L R}$ is being served by the serving-HLR. The MT is currently residing in serving-HLR. On move, the MT changes the RA and comes in the $\mathrm{RA}_{2}$. The $\mathrm{RA}_{2}$ is being served by the $\mathrm{VLR}_{4}$. This VLR4 is being served by the resident-HLR i.e. the MT is in its circle. In this move, the MT initiates the location registration process. Registration of the MT takes place at $V_{L R}$. The VLR ${ }_{4}$ informs the resident-HLR. The resident-HLR determines that the MT is coming from the serving-HLR. The resident-HLR sends location cancellation message to the serving-HLR. The serving-HLR sends this message to the $\mathrm{VLR}_{1}$. The VLR 1 deletes the entry of the MT and sends back an acknowledgement back to the serving-HLR. The serving-HLR sends an acknowledgement to the resident-HLR along with the MT profile. As the MT is in its circle, the resident-HLR ignores the MT profile and sends an acknowledgement back to the $\mathrm{VLR}_{4}$.

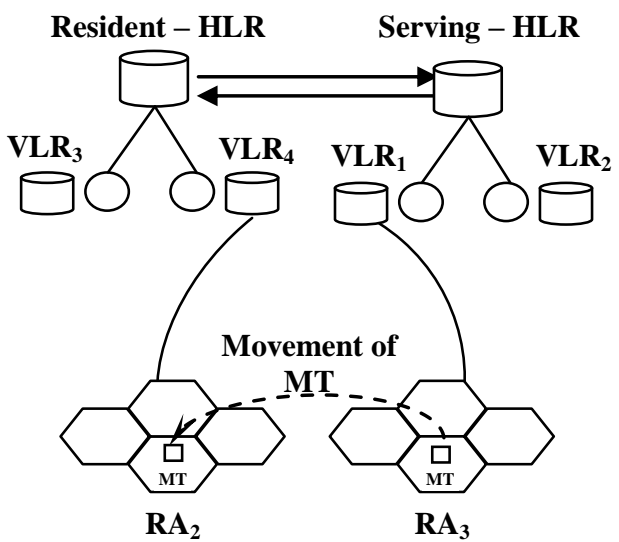

Fig 12: Inter-VLR-Inter-HLR move (from servingHLR to resident-HLR)

\subsubsection{MT moves from one serving-HLR to other serving-HLR}

This type of move is shown in the figure 13. The MT is residing in the $\mathrm{RA}_{4}$ of the $\mathrm{VLR}_{4}$ in the serving-HLR 1 . On move the MT comes to the $\mathrm{RA}_{5}$ of the $\mathrm{VLR}_{5}$ of serving$\mathrm{HLR}_{2}$. Registration of MT takes place at VLR . The VLR $_{5}$ informs serving-HLR ${ }_{2}$. Serving-HLR ${ }_{2}$ determines the resident-HLR of the MT and informs about this change. The resident-HLR has the information that MT is in serving-HLR ${ }_{1}$. The resident-HLR sends registration cancellation message to the serving-HLR ${ }_{1}$. The serving$\mathrm{HLR}_{1}$ sends registration cancellation message to the $\mathrm{VLR}_{4}$. The $\mathrm{VLR}_{4}$ deletes the MT profile and informs the serving$H_{L R}$. The Serving-HLR ${ }_{1}$ sends back an acknowledgement along with the MT profile to resident-HLR. The residentHLR has nothing to do with the profile. The resident-HLR sends back acknowledgement to the serving-HLR $\mathrm{H}_{2}$ along with the MT profile. The Serving-HLR ${ }_{2}$ sends back an acknowledgment to the $\mathrm{VLR}_{5}$. 

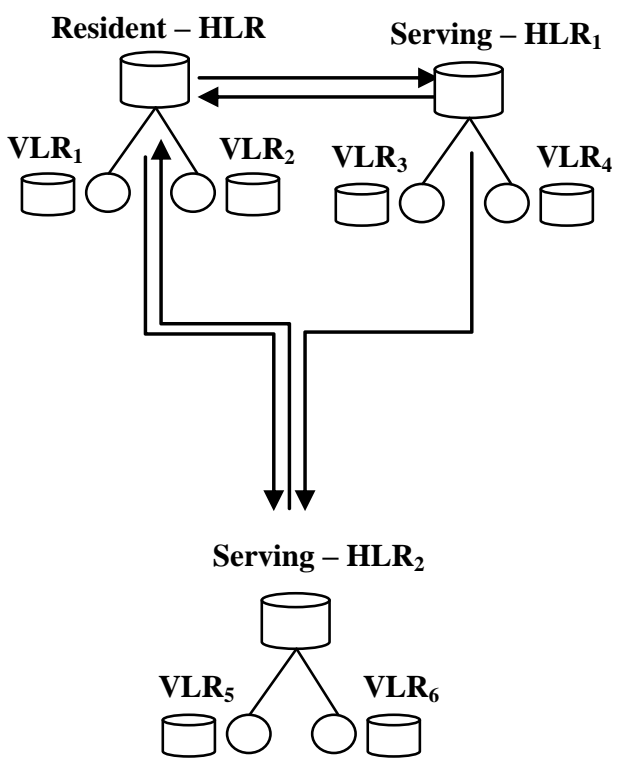

Fig 13: Inter-VLR-Inter-HLR move

\section{PERFORMANCE ANALYSIS}

An analytical model to evaluate the performance of explicit deregistration scheme is given in figure 14 .

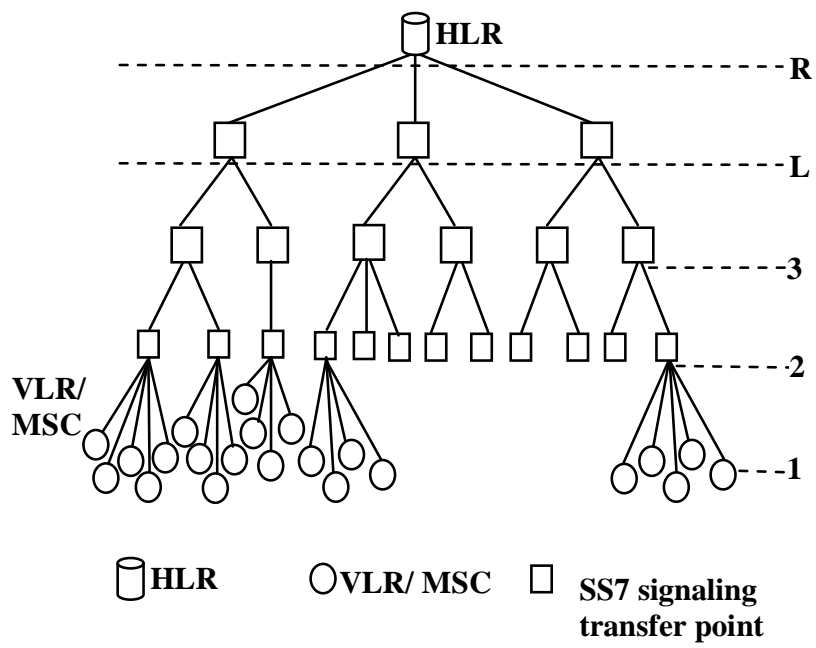

\section{Fig 14: An Analytical Model of HLR/VLR Architecture}

In this analysis, a hierarchical tree of $\mathrm{R}$ layers is used, as shown in Fig. 15. The layer R contains the root node and the layer 1 contains the leaf nodes. A database is installed on each node of the tree and the MTs are assigned to the leaf nodes. In the HLRVLR scheme, the network database, HLR, is situated on the only node of layer $\mathrm{R}$ and the VLRs are installed on the leaf nodes. The following terms are used in performance analysis:-

The following terms are used in performance analysis:-

$\mathbf{m}_{\mathbf{x}, \mathbf{y}}$ Layer of the closest common node to $\mathrm{RA}_{\mathrm{x}}$ and $\mathrm{RA}_{\mathrm{y}}$.

p Probability that the MT move is intra-VLR.

q Probability that the called and the calling MTs are served by the same VLR.

n New RA of the MU.

a Old RA of the MU. s RA of the calling unit (source).

d RA of the called MU (destination).

$\mathrm{P}\left(\mathbf{m}_{\mathbf{x}, \mathbf{y}}=\mathbf{i}\right)$ is defined as the probability that the closest common node to $\mathrm{RA}_{\mathrm{x}}$ and $\mathrm{RA}_{\mathrm{y}}$ is in layer $\mathrm{i}$. This probability can be given by the following equation.

$$
\begin{aligned}
P\left(m_{a, n}=i\right)= & p(1-p)^{i-1} \text { for } i=1,2 \ldots \ldots \ldots R-1 \\
& (1-p)^{i-1} \text { for } i=R \ldots \ldots \ldots \ldots \ldots(1) \\
P\left(m_{s, d}=i\right)= & q(1-q)^{i-1} \text { for } i=1,2 \ldots \ldots \ldots R-1 \\
& (1-q)^{i-1} \text { for } i=R \ldots \ldots \ldots \ldots \ldots . .(2)
\end{aligned}
$$

We furthermore denote the costs of various operations used in this analysis as follows:

$\mathbf{T}(\mathrm{i}, \mathrm{j})$ : Cost of transmitting a message over a link between two adjacent layers $\mathrm{i}$ and $\mathrm{j}$.

$\mathbf{C}_{\mathbf{m}}$ (i): Cost of accessing or updating a database in layer i.

\subsection{PERFORMANCE ANALYSIS OF EXPLICIT DE-REGISTRATION SCHEME AND IMPLICIT DE-REGISTRATION SCHEME}

In this analysis following terms have the significance as follows $[1,2,5$, and 6]:

$\boldsymbol{M}_{H L R-V L R(\text { explicit })}=$ Location Update Cost in Conventional HLR-VLR Architecture using Explicit De-registration Scheme.

$M_{H L R-V L R(i m p l i c i t)}=$ Location Update Cost in Conventional HLR-VLR Architecture using Implicit De-registration Scheme.

The estimated cost of the location update in PCS network by using explicit de-registration scheme is given below in Eq. No. 3.

$$
\begin{aligned}
M_{H L R-V L R(\text { explicit })} & =\left[P\left(m_{a, n}=1\right) \times C_{m}(1)\right] \\
& +\sum_{i=2}^{R} P\left(m_{a, n}=i\right) \\
& \times\left\{2 \times C_{m}(1)+C_{m}(R)\right. \\
& +4 T(1, R)\} \ldots \ldots(3)
\end{aligned}
$$

The first part of Eq. No. 3 is the cost of location update in intraVLR move. The second part illustrates the scenario after an inter-VLR move. $\mathrm{T}(1, \mathrm{~L})=\mathrm{T}(1,2)+\mathrm{T}(2,3)+\ldots+\mathrm{T}(\mathrm{L}-1, \mathrm{~L})$ is equal to the cost of traversing links between a node of layer 1(i.e., VLR) and the node of layer R (i.e., where an HLR is located). This cost is multiplied by 4 because, when a signaling message is sent from a VLR to the HLR, the latter sends a similar message to the old VLR. By adding the cost of the acknowledgment from the old VLR to the HLR and then from the HLR to the current VLR, we can justify the $4 \mathrm{~T}(1, \mathrm{~L})$.

The estimated cost of the location update in PCS network by using implicit de-registration scheme is given below in Eq. No. 4. 
$M_{H L R-V L R(\text { implicit })}$

$$
\begin{aligned}
& =\left[P\left(m_{a, n}=1\right) \times C_{m}(1)\right] \\
& +\sum_{i=2}^{R} P\left(m_{a, n}=i\right) \\
& \times\left\{C_{m}(1)+C_{m}(R)\right. \\
& +2 T(1, R)\} \ldots \ldots(4)
\end{aligned}
$$

The first part of above Eq. No. 4 is same as previous one, the second part illustrates the scenario after an inter-VLR move. $\mathrm{T}(1, \mathrm{~L})$ is estimated in same manner but this cost is multiplied by 2 because, we are not sending any request to old VLR for deregistration separately nor receiving any acknowledgement.

\subsubsection{Result}

In this section the numerical values of explicit and implicit deregistration schemes are evaluated and compared. Fig 15 and 16 show the performance of location update schemes with $R=5$ and $R=3$ respectively. In the figures, users are classified with respect to their moves. When $p$ (probability value) is very small, the mobile unit moves are not local.

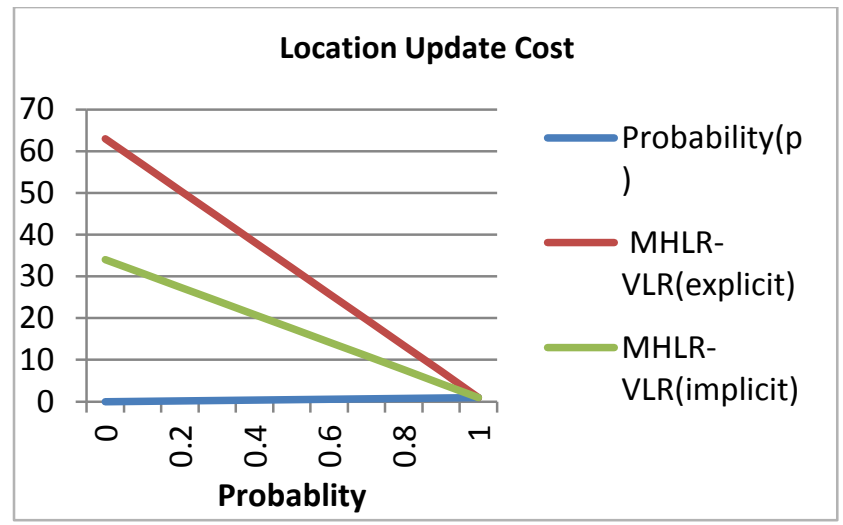

Fig15: Location Update Cost When $R=5$.

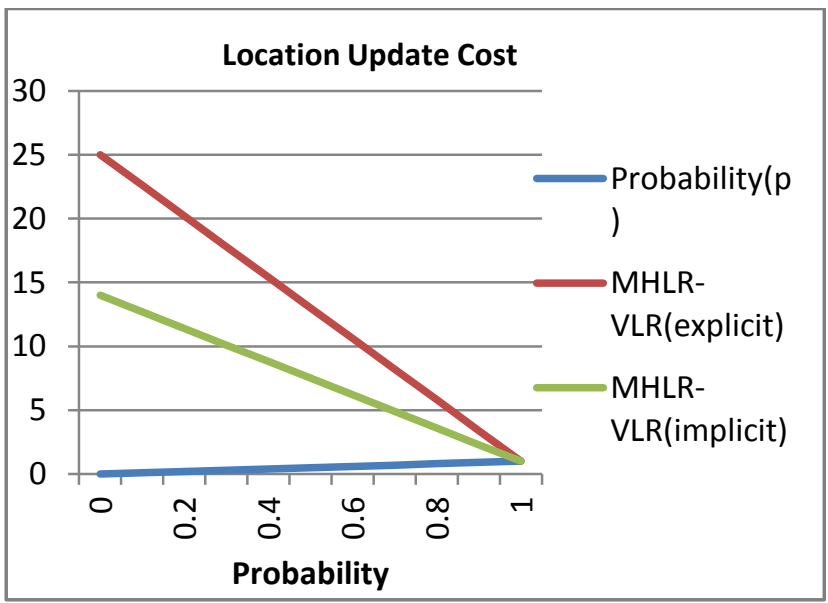

Fig16: Location Update Cost When $\mathbf{R}=3$.

\subsubsection{Conclusion}

Numerical values show that implicit de-registration scheme is cost wise efficient than the explicit de-registration scheme. However in implicit de-registration scheme, stale entries are at the VLR side. To remove the stale entries from the VLR side, three de-registration scheme is being used as: polling de- registration, timeout de-registration and group de-registration schemes. In the next sub section these schemes are being compared.

\subsection{PERFORMANCE ANALYSIS OF POLLING, TIMEOUT AND GROUP DE- REGISTRATION SCHEMES WITH IMPLICIT SCHEME: DE-REGISTRATION}

In this analysis following terms have the significance as follows [2]:

Estimated cost of location update with polling de-registration scheme is given as follows:

$\boldsymbol{M}_{\boldsymbol{H} L \boldsymbol{R}-\boldsymbol{V} \boldsymbol{L R}(\text { polling })}=$ Location Update Cost When Polling Deregistration is being used with Implicit De-registration Scheme.

$M_{\text {HLR-VLR(timeout })}=$ Location Update Cost When Timeout Deregistration is being used with Implicit De-registration Scheme. $\boldsymbol{M}_{H L R-V L R(\text { group })}=$ Location Update Cost When Group Deregistration is being used with Implicit De-registration Scheme.

$$
\begin{aligned}
M_{H L R-V L R(p o l l i n g)} & =\left[P\left(m_{a, n}=1\right) \times C_{m}(1)+2 \times 1\right] \\
& +\sum_{i=2}^{R} P\left(m_{a, n}=i\right) \\
& \times\left\{2 \times C_{m}(1)+C_{m}(R)+2 T(1, R)\right. \\
& +2 \times 1\} \ldots .(5)
\end{aligned}
$$

The first part of this Eq. No.5 is the cost of location update in intra-VLR move. We have added 2 because VLR is transmitting polling signals to its associated RAs and MTs are sending back acknowledgements the VLR.

In analytical model, VLR and MT are at same layer T $(1,1)$ becomes 1 . The link between VLR and MT is traversed twice so we get a cost 2 . The second part shows the scenario after an inter-VLR move. $\mathrm{T}(1, \mathrm{~L})$ is estimated in the same manner but this cost is multiplied by 2 because, we are not sending any deregistration request to the old VLR and no acknowledgement is being received in this response. If MT resides in an RA for some times then MT will follow the polling de-registration scheme and hence Cm (1) $+2 x 1$ is added.

Estimated cost of location update with timeout de-registration scheme is given as follows:

The first part of this Eq. No.6 is the cost of location update in intra-VLR move. We have added 1 because MTs are periodically sending registration message to the VLR. As VLR and MT are at same layer hence this cost is 1 . The second part shows the scenario after an inter-VLR move. $\mathrm{T}(1, \mathrm{~L})$ is estimated in the same manner but this cost is multiplied by 2 because, we are not sending any de-registration request to the old VLR and no acknowledgement is being received in response. If MT resides in an RA for some times then MT will follow the timeout de-registration scheme and hence $\mathrm{Cm}(1)+1$ is added. 


$$
\begin{aligned}
M_{\text {HLR-VLR(timeout })} & =\left[P\left(m_{a, n}=1\right) \times C_{m}(1)+1\right] \\
& +\sum_{i=2}^{R} P\left(m_{a, n}=i\right) \\
& \times\left\{2 \times C_{m}(1)+C_{m}(R)+2 T(1, R)\right. \\
& +1\} \ldots \ldots(6)
\end{aligned}
$$

Estimated cost of location update with group de-registration scheme is given as follows:

$$
\begin{aligned}
M_{H L R-V L R(\text { group })}= & {\left[P\left(m_{a, n}=1\right) \times C_{m}(1)\right]+\sum_{i=2}^{R} P\left(m_{a, n}=i\right) } \\
& \times\left\{2 \times C_{m}(1)+3 \times C_{m}(R)\right. \\
& +2 T(1, R)\} \ldots \ldots .(7)
\end{aligned}
$$

The first part of this Eq. No.7 is the cost of location update in intra-VLR move. When an MT leaves its RA and enters into new RA the new VLR sends a registration request to the HLR. HLR keeps the identification of the MT into the OML of the old VLR. After performing the MT's profile update by accessing its database HLR sends the acknowledgement message along with the OML of new VLR.

We see that HLR database is being consulted three times. The first access is done for putting the MT's identification into the old VLR's OML, second time for updating the MT's current location information and third time for emptying the OML of new VLR, further the entries of this OML is sent back with the acknowledgement. At the VLR side database is being consulted twice, first for the registration of new MT and second for deregistration of the entries sent by the HLR.

\subsubsection{Result}

In this section the numerical values of polling, timeout and group de-registration schemes with explicit HLR/VLR schemes are evaluated and compared. Fig 17 and 18 show the performance of location update schemes with $R=5$ and $R=3$ respectively. In the figures users are classified with respect to their moves. When $p$ (probability value) is very small, the mobile unit moves are not local. When probability $\mathrm{p}$ is 1 , it indicates that the MT is not performing any movement. It is case of intra-VLR move, in which the MT is not leaving the RA. When $p$ tends to zero, it indicates that the MT is performing inter-VLR move and so the MT is exhibiting high mobility.

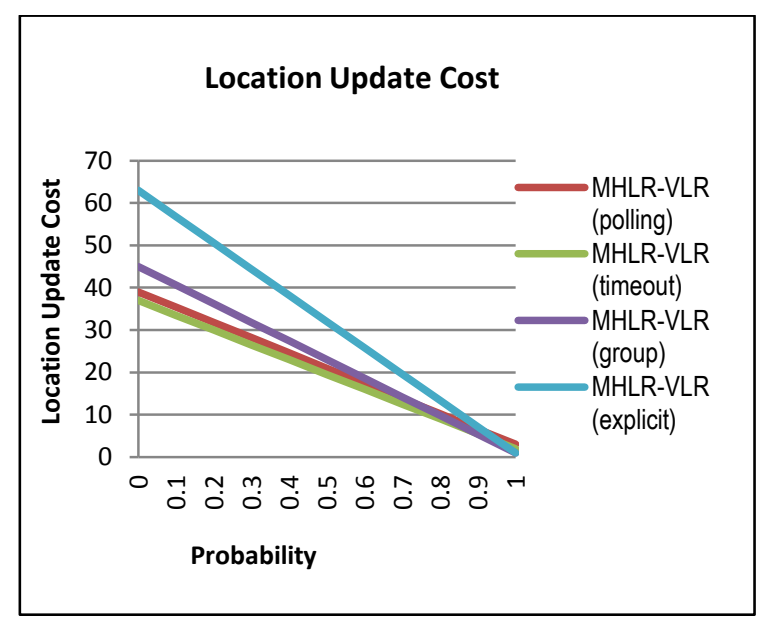

Fig.17: Location update cost when $R=5$

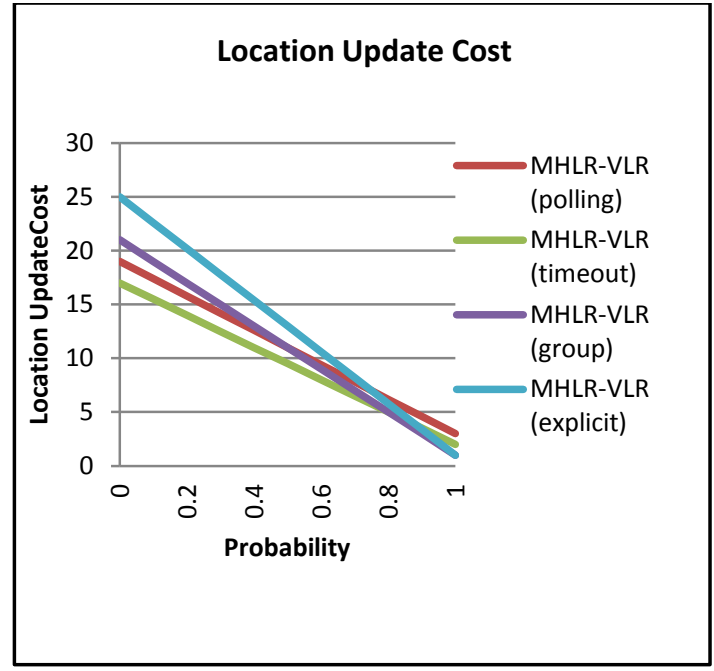

Fig.18: Location update cost when $R=3$

\subsubsection{Conclusion}

Results show that when implicit de-registration scheme is implement with timeout de-registration schemes stale entries are removed by consuming lesser cost than polling and group deregistration schemes. We have concluded group de-registration scheme as the best among the three because polling and timeout de-registration schemes suffer from the problem of synchronization. Group de-registration schemes removes the stale entries based on mobility from the VLR.

As stated earlier single HLR architecture suffers from the problem of bottleneck and during busy hours call misrouting may take place. To solve this problem of bottleneck, multi-HLR architecture is being proposed. Analysis done in the last section inspires to implement the group de-registration scheme instead of explicit de-registration scheme in the proposed multi HLR architecture. In the next sub section performance analysis of multi-HLR architecture is evaluated by implementing both explicit de-registration scheme and group de-registration scheme.

\subsection{PERFORMANCE ANALYSIS OF MULTI HLR ARCHITECTURE WITH GROUP DE-REGISTRATION SCHEME}

Proposed multi HLR architecture consists of many conventional HLR-VLR architecture zone wise or circle wise. All the HLRs of different circles are at the layer $\mathrm{R}$ and the VLRs are at the leaves of the tree as discussed in previous sections [3].

In this analysis we have defined three probabilities as:

a Probability of an MT to reside in its resident-HLR.

$\boldsymbol{\theta}$ Probability of an MT to reside in first serving-HLR.

及 Probability of an MT to reside in second serving-HLR.

Where,

$\theta=\beta=(1-\alpha)$

$\boldsymbol{M}_{\text {multiHLR-VLR(explicit })}=$ Location Update Cost in multi-HLR architecture when explicit de-registration scheme is used.

$\boldsymbol{M}_{\text {multiHLR-VLR(group) }}=$ Location Update Cost in multi-HLR architecture when group de-registration scheme is used. 
Rests of the terms are same as in the previous sections. Using Eq. No. (3) \& (7) we are deriving the expressions for location management schemes for multi HLR-VLR architectures as:

$$
\begin{aligned}
M_{\text {multiHLR-VLR(explicit })} & \\
& =\alpha x M_{H L R-V L R(\text { explicit })} \\
& +\beta x\left[M_{H L R-V L R(\text { explicit })}+2\right] \\
& +\theta x\left[M_{H L R-V L R(\text { explicit })}+4\right]
\end{aligned}
$$

$M_{\text {multiHLR-VLR(group) }}$

$$
\begin{aligned}
& =\alpha x M_{H L R-V L R(\text { group })} \\
& +\beta x\left[M_{H L R-V L R(\text { group })}+2\right] \\
& +\theta x\left[M_{H L R-V L R(\text { group })}+4\right]
\end{aligned}
$$

First part of equ. (10) shows the cost when the MT is always residing in resident-HLR. It means either MT is performing no movement or if movement is being performed it may be either inter-VLR or intra-VLR move in the resident area. In second part MT may perform either inter-VLR or intra-VLR movement in the roaming (outside the resident-HLR). A sum 2 is being done because of MT movement in between resident-HLR and serving-HLR. Both the HLRs are at same layer and hence one way traversal cost is 1 . Link traversal (in between resident and serving HLR) is being done twice hence 2. The third part of the equation shows the case when MT is coming to another serving-HLR by leaving the old serving-HLR. Link traversal between both serving-HLR and resident-HLR is being made 4 times as hence a sum of 4 is being added (for third case of interVLR-inter-HLR move). Explanation of Eq. No.(11) is same as (10) besides the fact that in this group de-registration is being used.

\subsubsection{Results}

In this section the numerical values of explicit de-registration scheme and group de-registration scheme implemented in the multi HLR-VLR architecture are evaluated and compared. Fig 19 and 20 show the performance of location update schemes with $\mathrm{R}=5$ and $\mathrm{R}=3$ respectively. In the figure $21 \& 22$, relative cost of group and explicit de-registration scheme when implemented in multi HLR architecture in percentage $\left(\left(\left(\mathrm{M}_{\text {multiHLR-VLR (group) }} /\left(\mathrm{M}_{\text {multiHLR-VLR (explicit) }}\right) * 100\right)\right.\right.$ are shown in the figure 23 , when $R=5$ and 3 .

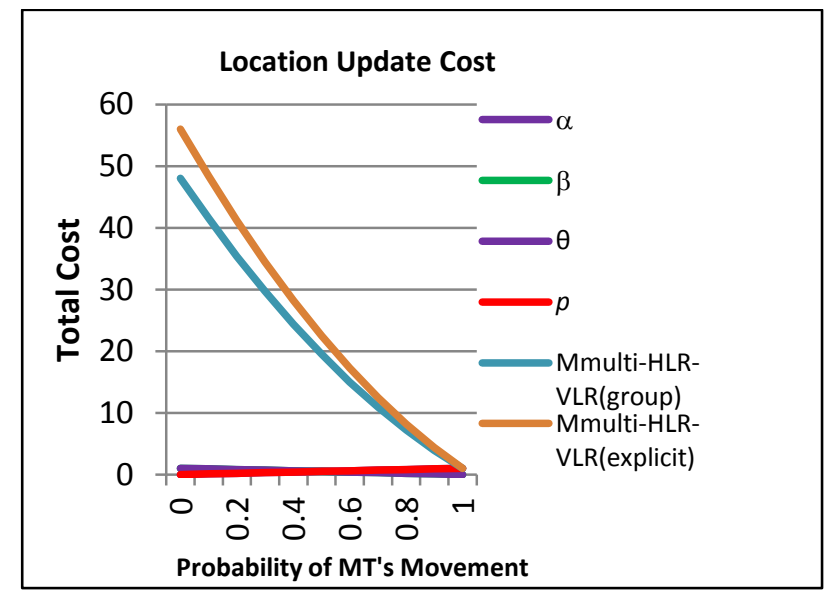

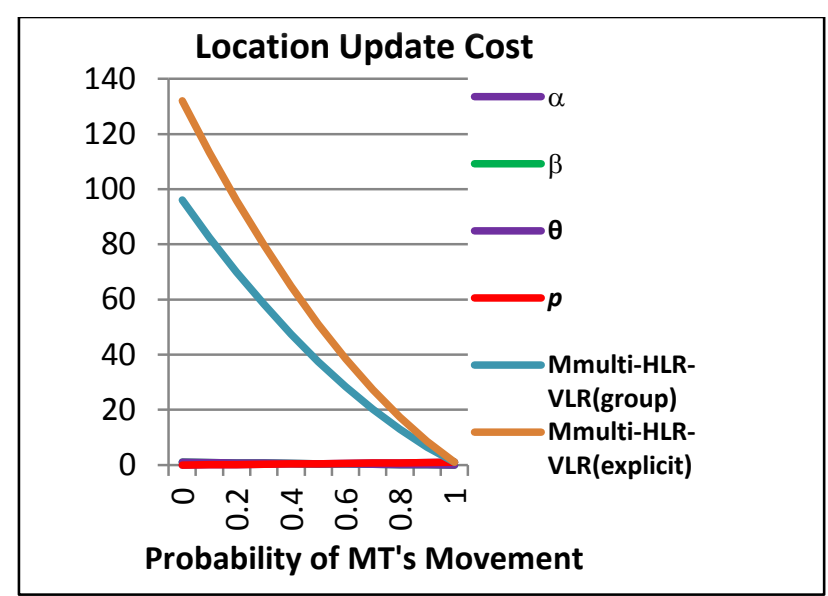

Fig 20: Location update cost when $R=3$.

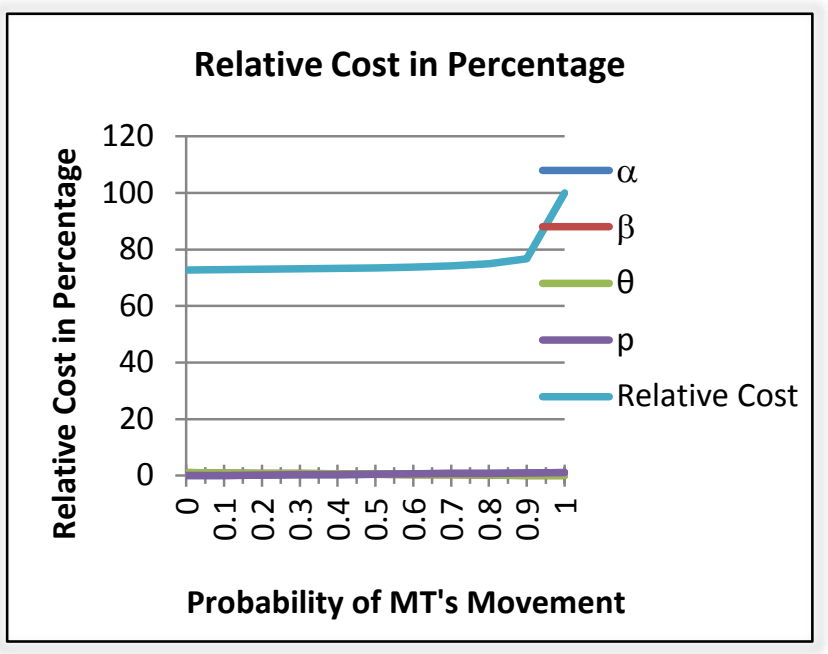

Fig 21: Relative location update cost when $R=5$.

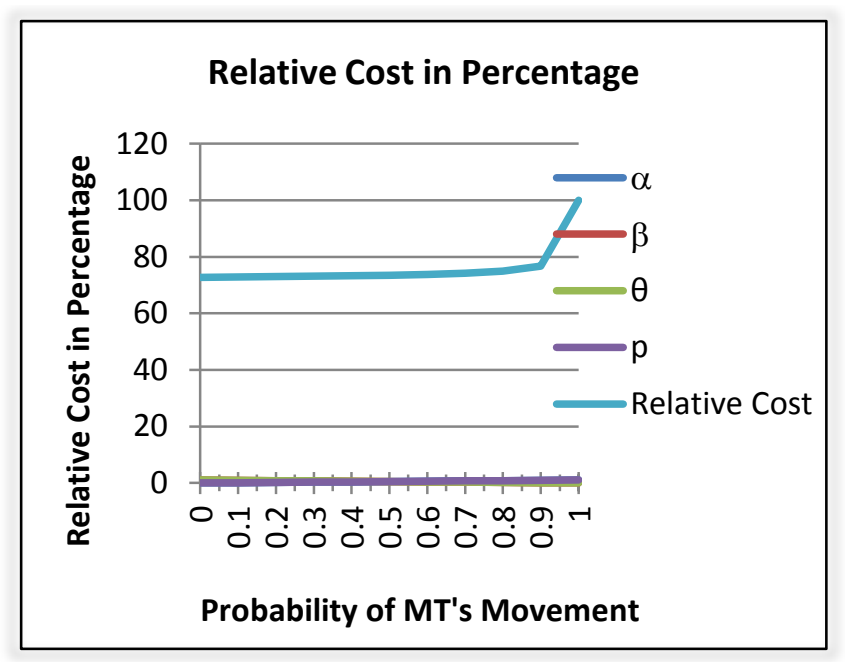

Fig 22: Relative location update cost when $R=3$.

Fig 19: Location update cost when $R=5$. 


\section{RESULTS}

In the last section results shows that implicit de-registration is cost wise efficient than the explicit de-registration scheme. But this implicit de-registration suffers from the problem of stale entries of the left MTs. To remove the stale entries from the VLR side three de-registration schemes are evaluated. On the basis of results and pros and cons associated with these deregistration schemes (polling, timeout and group) we have concluded that group de-registration scheme is the best one to implement with the multi-HLR architecture. Probability $p$ is showing the probability of intra-VLR move. This intra-VLR move may take place either in resident-HLR or in the servingHLR. When its value is 1 , it indicates that MT is not performing any movement. When this $p$ tends to 0 , it indicates high mobility in the RAs of the network in single HLR architecture. In case of multi-HLR architecture the MT may be either in the resident-HLR or in the serving-HLR (in roaming) if $p$ is tending to 0 it indicates that MT is performing high mobility (high mobility means, inter-VLR move is being performed very frequently). Probability, $\alpha$ shows the probability of an MT to reside in the resident-HLR. If this probability is 1 , the MT is always in the resident-HLR, the MT's movement may be either intra-VLR or inter-VLR in this region. When $\alpha$ becomes 1 , the MT is always in roaming. When $\alpha$ and $p$ equal to 0 , it shows the maximum degree of movement, in this case group deregistration scheme is $72.73 \%$ efficient than the conventional explicit de-registration scheme when $\mathrm{R}=5$ and $85.71 \%$ in case when $\mathrm{R}=3$.

\section{CONCLUSION}

Multi HLR architecture is bottleneck free. This architecture is centralized in nature. Dividing the entire network in zone wise or circle wise reduces the load of HLR. On the basis of the analysis made in the previous section we can conclude that possibility of call misrouting is less in multi HLR architecture in comparison to single HLR architecture. A better way of call delivery scheme is being proposed as: If a call is being initiated by an MT in the network, instead of querying HLR for the call routable path, first the VLR from which the MT is calling should be checked. During movement there is a chance that both calling and called MTs are residing in the same RA. This is a heuristic even though it is applicable in case of roaming. If a miss occur both in the VLR and in the serving HLR, residentHLR is consulted to get the actual location of the MT.

\section{REFERENCES}

[1] Rajeev R. Kumar Tripathi, Sudhir Agrawal and Swati Tiwari, "Performance Analysis of De-registration Strategy in Personal Communication Network," IJCA, vol.24-no.1, June 2011.

[2] Rajeev R. Kumar Tripathi, Sudhir Agrawal and Swati Tiwari, "Modified HLR-VLR Location Management Scheme in PCS Network," IJCA, vol.6-no.5, Sept 2010.

[3] Rajeev R. Kumar Tripathi, G.S. Chandel and Ravindra Gupta, "Multi HLR Architecture for Improving Location Management in PCS Network,"IJCA, vol.51, no.20, Aug.2012.

[4] Z. Mao, C. Douligeris, "A novel deregistration strategy for mobile network," IEEE VTS 2750-2754, vol.6, Aug 2002.

[5] Haider Safa and Samuel Pierre, "A New Architecture for Improving Location management in PCS Network," Journal of Computer Science1 (2):249-258, 2005.

[6] Sarvpal H. Singh, Sudhir Agrawal and Vijay Kumar "Performance Evaluation of Location Management Techniques in PCS Networks," IJCA, vol.15, no-8, Feb2011.

[7] Guan-Chi Chen and Suh-Yin Lee, "Evaluation of Distributed and Replicated HLR for Location Management in PCS Network," Journal Of Information Science And Engineering 19, 85-101 (2003).

[8] A. Bar-Noy, I. Kessler and M. Sidi, "Mobile Users: To Update or Not to Update?" ACM-Baltzer J. Wireless Networks, vol. 1, no. 2, July 1995, pp. 175p;86.

[9] Sarvpal H. Singh, Vijay Kumar, "A Modified Multi-HLR Architecture for Implicit De-registration Strategies", IJCA, vol.52, n0.13, Aug.2012.

[10] Li, Pan and Xiao, "A Dynamic HLR Location Management Scheme for PCS Network", IEEE, INFOCOM, 2004.

[11] Akyildiz, I.F., W. Wang, 2002. A dynamic location management scheme for next-generation multi-tier pcs systems. IEEE Transactions Wireless Communications, 1: 178-189 